Commun. Fac. Sci. Univ. Ank. Ser. A1 Math. Stat.

Volume 69, Number 1, Pages 684-698(2020)

DOI: $10.31801 /$ cfsuasmas.487789

ISSN 1303-5991 E-ISSN 2618-6470

http://communications.science.ankara.edu.tr/index.php?series=A1

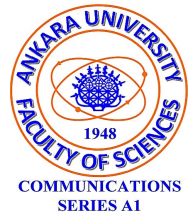

\title{
CURVES AND RULED SURFACES ACCORDING TO ALTERNATIVE FRAME IN DUAL SPACE
}

\author{
SÜLEYMAN ŞENYURT AND ABDUSSAMET ÇALIŞKAN
}

\begin{abstract}
In this paper, the vectorial moments of the alternative vectors are expressed in terms of altrenative frame. According to the new versions of these vectorial moments, the parametric equations of the closed ruled surfaces corresponding to the $(\widehat{N}),(\widehat{C}),(\widehat{W})$ dual curves are given. The integral invariants of these surfaces are computed and illustrated by presenting with examples.
\end{abstract}

\section{IntRODUCTION}

There are many studies on the classical differential geometry of curve and surface theories and are still being studied. A ruled surface in $I R^{3}$ is a surface which contains at least one 1-parameter family of straight lines. Thus a ruled surface has a parametrization in the form

$$
\vec{\varphi}(s, v)=\vec{\alpha}(s)+v \vec{x}(s)
$$

where we call $\alpha$ the anchor curve and the generator vector $x$ as ruled surface. When the above ruled surface satisfies $\varphi(s+2 \pi, v)=\varphi(s, v)$ it is called closed ruled surface. The properties of the ruled surface obtained according to the condition of the anchor curve or the generator vector are available in the books of differential geometry, [1, 2, 13]. Bertrand offsets, Mannheim offsets and involute-evolute offsets are obtained when special curves such as Bertrand, Mannheim and involute-evolute are taken as base curves. The geometric properties of these curves and surfaces are available in some references, 8, , 9, 10, 12, 14, 15, 16.

If the vectorial moment of the $x$ vector is denoted by $x^{*}$, then $x^{*}=\alpha \wedge x$. If $X$ has the norm $\|X\|=1$, then it is dual point on the dual unit sphere. According to E.Study theorem, there exists a one-to-one transformation between the dual points on the unit dual sphere and the oriented lines in $I R^{3}$. A one-parameter set of points (a dual curve) on dual unit sphere corresponds to a one-parameter family

Received by the editors: November 26, 2018; Accepted: January 14, 2020.

2010 Mathematics Subject Classification. 14H45, 14H50, 53A04.

Key words and phrases. Alternative frame, closed ruled surface, vectorial moment, distribution parameter, Gauss curvature, dual angle of pitch, viviani's curve.

(C) 2020 Ankara University Communications Faculty of Sciences University of Ankara-Series A1 Mathematics and Statistics 
of oriented lines in $E^{3}$, which defines a ruled surface. This dual curve is called the dual spherical image of the ruled surface, [5, 7.

The dual expression of a ruled surface in (1) is

$$
\varphi(s, u)=\vec{x}(s) \wedge \vec{x}^{*}(s)+u \vec{x}(s)
$$

where the $\vec{x}(s) \wedge \vec{x}^{*}(s)$ is the anchor curve. $s$ is not the arc-parameter of this curve [5, 17. The dual angle of pitch of the closed ruled surface in (2) is defined by [5]

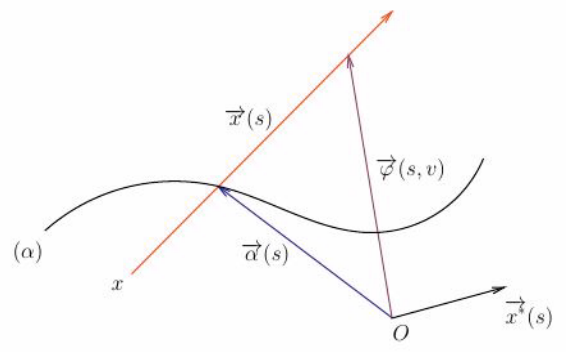

Figure 1. The dual expression of a ruled surface.

$$
\Lambda_{X}=-\langle D, X\rangle=\lambda_{x}-\varepsilon L_{x}
$$

Here, $\lambda_{x}$ and $L_{x}$ are real integral invariants [5].

Osman Gürsoy's study showed that the dual integral invariant of a closed ruled surface, the dual angle of pitch, corresponds to the dual spherical surface area described by the dual spherical indicatrix of the closed ruled surface. Further, geometric interpretations of the real angle of pitch and the real pitch of a closed ruled surface were given [6]. In [4, the pitch, the angle of pitch and the dual angle of pitch of closed ruled surface corresponding to a closed curve on dual unit sphere were investigated. In [17, a differential equation characterizing the dual spherical curves and an explicit solution of this differential equation was given. By investigating one parameter spherical motion in with two different kinds of dual indicatrice curves, Yaylı and Saraçoğlu obtained the ruled surfaces that correspond to tangent, principal normal and binormal indicatrices of the dual curve were developable, [20].

\section{Preliminaries}

In $E^{3}$, standard inner product is given by

$$
\langle x, x\rangle=x_{1}^{2}+x_{2}^{2}+x_{3}^{2}
$$

where $x=\left(x_{1}, x_{2}, x_{3}\right) \in E^{3}$. Let $\alpha: I \rightarrow E^{3}$ be a unit speed curve denote by $\{T, N, B\}$ the moving Frenet frame. $T(s)$ is the tangent vector field, $N(s)$ is the 
principal normal vector field and $B(s)$ is the binormal vector field of curve $\alpha(s)$, respectively. The Frenet formulas are given by [2]

$$
T^{\prime}(s)=\kappa(s) N(s), N^{\prime}(s)=-\kappa(s) T(s)+\tau(s) B(s), B^{\prime}(s)=-\tau(s) N(s) .
$$

Here curvature and torsion of the curve $\alpha(s)$ are defined with [2]

$$
\kappa(s)=\left\|\alpha^{\prime \prime}(s)\right\|, \tau(s)=\frac{\left\langle\alpha^{\prime}(s) \wedge \alpha^{\prime \prime}(s), \alpha^{\prime \prime \prime}(s)\right\rangle}{\| \alpha^{\prime}(s) \wedge \alpha^{\prime \prime 2}} .
$$

The vector $W$ is called unit Darboux vector and defined by 3 ]

$$
W=\frac{w}{\|w\|}=\frac{1}{\sqrt{\kappa^{2}+\tau^{2}}}(\tau T+\kappa B)
$$

It is obvious that the Darboux vector is perpendicular to the principal normal vector field $N$. If $C$ is taken as $C=W \wedge N$, then $\{N, C, W\}$ are another orthonormal moving frame along the curve $\alpha$. This frame is called an alternative frame. The derivative formulae of the alternative frame is given by

$$
\left[\begin{array}{l}
N^{\prime} \\
C^{\prime} \\
W^{\prime}
\end{array}\right]=\left[\begin{array}{ccc}
0 & \beta & 0 \\
\beta & 0 & \gamma \\
0 & -\gamma & 0
\end{array}\right]\left[\begin{array}{c}
N \\
C \\
W
\end{array}\right]
$$

where $\beta=\sqrt{\kappa^{2}+\tau^{2}}$ and $\gamma=\frac{\kappa^{2}}{\kappa^{2}+\tau^{2}}\left(\frac{\tau}{\kappa}\right)^{\prime}$.The relationship between the Frenet frame and alternative frame are

$$
\left\{\begin{array} { l } 
{ C = - \overline { \kappa } T + \overline { \tau } B } \\
{ W = \overline { \tau } T + \overline { \kappa } B }
\end{array} \text { or } \quad \left\{\begin{array}{l}
T=-\bar{\kappa} C+\bar{\tau} W \\
B=\bar{\tau} C+\bar{\kappa} W
\end{array}\right.\right.
$$

where principal normal vector $N$ is same in both frames, $\bar{\kappa}=\frac{\kappa}{\beta}$ and $\bar{\tau}=\frac{\tau}{\beta}$, [11, 19].

Let $f(s), g(s)$ and $h(s)$ be al least $C^{3}$ - functions. $\alpha(s)$ can be written in the form of

$$
\alpha(s)=f(s) T(s)+g(s) N(s)+h(s) B(s)
$$

as a linear combination of the Frenet vectors $\{T, N, B\}$, 18. By differentiating both side of $(10)$, it is obtained [18]

$$
f^{\prime}(s)-g(s) \kappa(s)=1, h^{\prime}(s)+g(s) \tau(s)=0, g^{\prime}(s)+f(s) \kappa(s)-h(s) \tau(s)=0 .
$$

\section{Curves and Ruled Surfaces According to Alternative Frame in Dual Space}

The geometric location of $\widehat{N}=N+\varepsilon N^{*}, \widehat{C}=C+\varepsilon C^{*}$ and $\widehat{W}=W+\varepsilon W^{*}$ vectors draws closed curves on the dual sphere. These closed curves are shown as $(\widehat{N}),(\widehat{C})$ and $(\widehat{W})$ respectively. According to Study's theorem these closed curves correspond to closed ruled surfaces. The dual expressions of the closed ruled surfaces corresponding to $(\widehat{N}),(\widehat{C})$ and $(\widehat{W})$ dual curves are

$$
\begin{aligned}
& \psi_{\widehat{N}}(s, v)=\beta_{N}(s)+v N(s), \beta_{N}(s)=N(s) \wedge N^{*}(s), \\
& \psi_{\widehat{C}}(s, v)=\beta_{C}(s)+v C(s), \beta_{C}(s)=C(s) \wedge C^{*}(s),
\end{aligned}
$$




$$
\psi_{\widehat{W}}(s, v)=\beta_{W}(s)+v W(s), \beta_{W}(s)=W(s) \wedge W^{*}(s) .
$$

It is known that the curve $\alpha$ is written in the form of Frenet vectors. Using the equations (9) and (10), we can write as the linear combination of alternative vectors as follows:

$$
\begin{aligned}
\alpha(s) & =f(-\bar{\kappa} C+\bar{\tau} W)+g N+h(\bar{\tau} C+\bar{\kappa} W) \\
& =g N+\frac{g^{\prime}}{\beta} C+\left(\frac{f \tau+h \kappa}{\beta}\right) W .
\end{aligned}
$$

Considering the above equation, vectorial moments of $N, C, W$ are given respectively

$$
\begin{aligned}
N^{*} & =\alpha \wedge N=\left(g N+\frac{g^{\prime}}{\beta} C+\left(\frac{f \tau+h \kappa}{\beta}\right) W\right) \wedge N \\
& =-\frac{g^{\prime}}{\beta} W+\left(\frac{f \tau+h \kappa}{\beta}\right) C, \\
C^{*} & =\alpha \wedge C=\left(g N+\frac{g^{\prime}}{\beta} C+\left(\frac{f \tau+h \kappa}{\beta}\right) W\right) \wedge C \\
& =g W-\left(\frac{f \tau+h \kappa}{\beta}\right) N, \\
W^{*} & =\alpha \wedge W=\left(g N+\frac{g^{\prime}}{\beta} C+\left(\frac{f \tau+h \kappa}{\beta}\right) W\right) \wedge W \\
& =\frac{g^{\prime}}{\beta} N-g C .
\end{aligned}
$$

Using (14) in 12, The dual expressions of the closed ruled surfaces corresponding to $(\widehat{N}),(\widehat{C})$ and $(\widehat{W})$ dual curves are given by

$$
\begin{aligned}
\psi_{\widehat{N}}(s, v) & =N \wedge N^{*}+v N=N \wedge\left(\frac{1}{\beta}\left((f \tau+h \kappa) C-g^{\prime} W\right)\right)+v N \\
& =\frac{1}{\beta}\left(g^{\prime} C+(f \tau+h \kappa) W\right)+v N \\
\psi_{\widehat{C}}(s, v) & =C(s) \wedge C^{*}(s)+v C(s)=C \wedge\left(-\frac{1}{\beta}(f \tau+h \kappa) N+g W\right)+v C \\
& =\frac{1}{\beta}(f \tau+h \kappa) W+g N+v C \\
\varphi_{\widehat{W}}(s, v) & =W(s) \wedge W^{*}(s)+v W(s)=W \wedge\left(\frac{g^{\prime}}{\beta} N-g C\right)+v W(s) \\
& =g N+\frac{g^{\prime}}{\beta} C+v W(s) .
\end{aligned}
$$


Theorem 1. Distribution parameters of the closed ruled surfaces corresponding to the $(\widehat{N}),(\widehat{C}),(\widehat{W})$ dual curves are given by

$$
\begin{aligned}
& P_{\widehat{N}}=\frac{1}{\beta}\left(-\left(\frac{f \tau+h \kappa}{\beta}\right)^{\prime}-\frac{\gamma}{\beta} g^{\prime}\right), \\
& P_{\widehat{C}}=\frac{g^{\prime} \gamma+\beta\left(\frac{1}{\beta}(f \tau+h \kappa)\right)^{\prime}}{\beta^{2}+\gamma^{2}}, \\
& P_{\widehat{W}}=0 .
\end{aligned}
$$

Proof. We know that the distribution parameter of the closed ruled surface corresponding to the $(\widehat{N})$ dual curve is calculated by

$$
P_{\widehat{N}}=\frac{\operatorname{det}\left(\left(N \wedge N^{*}\right)^{\prime}, N, N^{\prime}\right)}{\| N^{\prime 2}}
$$

It can be written that

$$
\begin{aligned}
\left(N \wedge N^{*}\right)^{\prime}= & \left(\frac{1}{\beta}\right)^{\prime}\left(g^{\prime} C+(f \tau+h \kappa) W\right)+\frac{1}{\beta}\left(g^{\prime \prime} C+g^{\prime}(-\beta N+\gamma W)\right. \\
& \left.+(f \tau+h \kappa)^{\prime} W-\gamma(f \tau+h \kappa)\right) C \\
= & -g^{\prime} N+\left(\left(\frac{g^{\prime}}{\beta}\right)^{\prime}-\frac{\gamma}{\beta}(f \tau+h \kappa)\right) C+\left(\left(\frac{f \tau+h \kappa}{\beta}\right)^{\prime}+\frac{\gamma}{\beta} g^{\prime}\right) W .
\end{aligned}
$$

If this value is substituted into (17), the following result is obtained

$$
\begin{aligned}
& P_{\widehat{N}}=\frac{1}{\beta^{2}}\left|\begin{array}{ccc}
-g^{\prime} & \left(\frac{g^{\prime}}{\beta}\right)^{\prime}-\frac{\gamma}{\beta}(f \tau+h \kappa) & -\left(\frac{f \tau+h \kappa}{\beta}\right)^{\prime}-\frac{\gamma}{\beta} g^{\prime} \\
1 & 0 & 0 \\
0 & \beta
\end{array}\right| \\
& =\frac{1}{\beta}\left(-\left(\frac{f \tau+h \kappa}{\beta}\right)^{\prime}-\frac{\gamma}{\beta} g^{\prime}\right) .
\end{aligned}
$$

Similarly, with the values of

$$
\begin{aligned}
\left(C \wedge C^{*}\right)^{\prime} & =g^{\prime} N+g N^{\prime}+\left(\frac{f \tau+h \kappa}{\beta}\right)^{\prime} W+\frac{1}{\beta}(f \tau+h \kappa) W^{\prime} \\
& =g^{\prime} N+\left(g \beta-\frac{\gamma}{\beta}(f \tau+h \kappa)\right) C+\left(\frac{1}{\beta}(f \tau+h \kappa)\right)^{\prime} W \\
\left(W \wedge W^{*}\right)^{\prime} & =g^{\prime} N+g N^{\prime}+\left(\frac{g^{\prime}}{\beta}\right)^{\prime} C+\frac{g^{\prime}}{\beta} C^{\prime}
\end{aligned}
$$




$$
=\left(g \beta+\left(\frac{g^{\prime}}{\beta}\right)^{\prime}\right) C+\frac{\gamma}{\beta} g^{\prime} W,
$$

distribution parameters of the closed ruled surfaces corresponding to the $(\widehat{C})$ and $(\widehat{W})$ dual curves are

$$
P_{\widehat{C}}=\frac{g^{\prime} \gamma+\beta\left(\frac{1}{\beta}(f \tau+h \kappa)\right)^{\prime}}{\beta^{2}+\gamma^{2}}, \quad P_{\widehat{W}}=0
$$

Theorem 2. Gauss curvatures of closed ruled surfaces corresponding to $(\widehat{N}),(\widehat{C})$ and $(\widehat{W})$ dual curves are

$$
\begin{aligned}
& K_{\widehat{N}}(P)=-\left(\frac{\left(\frac{\gamma}{\beta} g^{\prime}+\left(\frac{f \tau+h \kappa}{\beta}\right)^{\prime}\right) \beta}{\sqrt{\left(\left(\frac{g^{\prime}}{\beta}\right)^{\prime}-\frac{\gamma(f \tau+h \kappa)}{\beta}+v \beta\right)^{2}+\left(\frac{\gamma}{\beta} g^{\prime}+\left(\frac{f \tau+h \kappa}{\beta}\right)^{\prime}\right)^{2}}}\right)^{2}, \\
& K_{\widehat{C}}(P)=-\left(\frac{\beta\left(\frac{f \tau+h \kappa}{\beta}\right)^{\prime}+\gamma g^{\prime}}{\sqrt{\left(g^{\prime}-v \beta\right)^{2}+\left[\left(\frac{f \tau+h \kappa}{\beta}\right)^{\prime}+v \gamma\right]^{2}}}\right)^{2}, \\
& K_{\widehat{W}}(P)=0 .
\end{aligned}
$$

Proof. For the closed ruled surface $\psi_{\widehat{N}}(s, v)$, the partial derivative is taken according to $s$ and $v$, it is found

$$
\begin{aligned}
\psi_{\widehat{N}_{v}}(s, v)= & N \\
\psi_{\widehat{N}_{s}}(s, v)= & \left(\frac{g^{\prime}}{\beta} C+\left(\frac{f \tau+h \kappa}{\beta}\right) W\right)^{\prime}+v N^{\prime}(s) \\
= & -g^{\prime} N+\left(\left(\frac{g^{\prime}}{\beta}\right)^{\prime}-\frac{\gamma}{\beta}(f \tau+h \kappa)+v \beta\right) C \\
& +\left(\frac{\gamma}{\beta} g^{\prime}+\left(\frac{f \tau+h \kappa}{\beta}\right)^{\prime}\right) W .
\end{aligned}
$$

Taking into account that inner product, we compute

$$
\left\langle\psi_{\widehat{N}_{v}}(s, v), \psi_{\widehat{N}_{s}}(s, v)\right\rangle=-g^{\prime} \neq 0
$$


Using the Gram-Schmidt process, it can be seen that

$$
\begin{aligned}
y_{1} & =x_{1}=N \\
y_{2} & =-\frac{\left\langle y_{1}, x_{2}\right\rangle}{\left\langle y_{1}, y_{1}\right\rangle} y_{1}+x_{2} \\
& =\left(\left(\frac{g^{\prime}}{\beta}\right)^{\prime}-\frac{\gamma(f \tau+h \kappa)}{\beta}+v \beta\right) C+\left(\frac{\gamma}{\beta} g^{\prime}+\left(\frac{f \tau+h \kappa}{\beta}\right)^{\prime}\right) W \\
E_{1} & =\frac{y_{1}}{\left\|y_{1}\right\|}=N \\
E_{2} & =\frac{y_{2}}{\left\|y_{2}\right\|}=\frac{\left(\left(\frac{g^{\prime}}{\beta}\right)^{\prime}-\frac{\gamma(f \tau+h \kappa)}{\beta}+v \beta\right) C+\left(\frac{\gamma}{\beta} g^{\prime}+\left(\frac{f \tau+h \kappa}{\beta}\right)^{\prime}\right) W}{\sqrt{\left(\left(\frac{g^{\prime}}{\beta}\right)^{\prime}-\frac{\gamma(f \tau+h \kappa)}{\beta}+v \beta\right)^{2}+\left(\frac{\gamma}{\beta} g^{\prime}+\left(\frac{f \tau+h \kappa}{\beta}\right)^{\prime}\right)^{2}}} .
\end{aligned}
$$

For a closed ruled surface with parametrization $\psi_{\widehat{N}}(s, v)$, the normal vector is given by

$$
\begin{aligned}
N_{\widehat{N}}= & E_{1} \wedge E_{2} \\
= & \frac{-\left(\frac{\gamma}{\beta} g^{\prime}+\left(\frac{f \tau+h \kappa}{\beta}\right)^{\prime}\right) C+\left(\left(\frac{g^{\prime}}{\beta}\right)^{\prime}-\frac{\gamma(f \tau+h \kappa)}{\beta}+v \beta\right) W}{\sqrt{\left(\left(\frac{g^{\prime}}{\beta}\right)^{\prime}-\frac{\gamma(f \tau+h \kappa)}{\beta}+v \beta\right)^{2}+\left(\frac{\gamma}{\beta} g^{\prime}+\left(\frac{f \tau+h \kappa}{\beta}\right)^{\prime}\right)^{2}}} .
\end{aligned}
$$

On the other hand, we compute

$$
\begin{aligned}
S_{\widehat{N}}\left(E_{2}\right) & =D_{E_{2}} N_{\widehat{N}}=D_{\frac{y_{2}}{\left\|y_{2}\right\|}} N_{\widehat{N}}=\frac{1}{\left\|y_{2}\right\|} D_{\lambda y_{1}+x_{2}} N_{\widehat{N}}=\frac{1}{\left\|y_{2}\right\|}\left(\lambda \frac{\partial N_{\widehat{N}}}{\partial v}+\frac{\partial N_{\widehat{N}}}{\partial s}\right) \\
& \Rightarrow\left\langle S_{\widehat{N}}\left(E_{2}\right), E_{1}\right\rangle=\left(\frac{\left(\frac{\gamma}{\beta} g^{\prime}+\left(\frac{f \tau+h \kappa}{\beta}\right)^{\prime}\right) \beta}{\sqrt{\left(\left(\frac{g^{\prime}}{\beta}\right)^{\prime}-\frac{\gamma(f \tau+h \kappa)}{\beta}+v \beta\right)^{2}+\left(\frac{\gamma}{\beta} g^{\prime}+\left(\frac{f \tau+h \kappa}{\beta}\right)^{\prime}\right)^{2}}}\right),
\end{aligned}
$$

where $\lambda=-\frac{\left\langle y_{1}, x_{2}\right\rangle}{\left\langle y_{1}, y_{1}\right\rangle}$. Since shape operator is self-adjoint, we can write $\left\langle S\left(E_{2}\right), E_{1}\right\rangle=$ $\left\langle S\left(E_{1}\right), E_{2}\right\rangle$. If the main direction of the surface is the asymptotic direction, the shape operator is $\left\langle S\left(E_{1}\right), E_{1}\right\rangle=0$, [1]. Then, Gauss curvature of closed ruled surface $\psi_{\widehat{N}}(s, v)$ is

$$
K_{\widehat{N}}(P)=\operatorname{det}\left(S_{P}\right)=\left[\begin{array}{ll}
\left\langle S\left(E_{1}\right), E_{1}\right\rangle & \left\langle S\left(E_{1}\right), E_{2}\right\rangle \\
\left\langle S\left(E_{2}\right), E_{1}\right\rangle & \left\langle S\left(E_{2}\right), E_{2}\right\rangle
\end{array}\right]
$$




$$
=-\left(\frac{\left(\frac{\gamma}{\beta} g^{\prime}+\left(\frac{f \tau+h \kappa}{\beta}\right)^{\prime}\right) \beta}{\sqrt{\left(\left(\frac{g^{\prime}}{\beta}\right)^{\prime}-\frac{\gamma(f \tau+h \kappa)}{\beta}+v \beta\right)^{2}+\left(\frac{\gamma}{\beta} g^{\prime}+\left(\frac{f \tau+h \kappa}{\beta}\right)^{\prime}\right)^{2}}}\right)^{2} .
$$

Likewise, Gauss curvatures of closed ruled surfaces $\psi_{\widehat{C}}(s, v)$ and $\psi_{\widehat{W}}(s, v)$ are

$$
\begin{aligned}
& K_{\widehat{C}}(P)=-\left(\frac{\beta\left(\frac{f \tau+h \kappa}{\beta}\right)^{\prime}+\gamma g^{\prime}}{\sqrt{\left(g^{\prime}-v \beta\right)^{2}+\left[\left(\frac{f \tau+h \kappa}{\beta}\right)^{\prime}+v \gamma\right]^{2}}}\right)^{2}, \\
& K_{\widehat{W}}(P)=0 .
\end{aligned}
$$

Theorem 3. The instantaneous dual Pfaffian vector and the dual Steiner vector are given by respectively

$$
\widehat{w}=\tau T+\kappa B+\varepsilon\left(g \kappa T+g^{\prime} N-g \tau B\right)
$$

and

$$
D=d+\varepsilon d^{*}=T \oint \tau+B \oint \kappa+\varepsilon\left(T \oint g \kappa+N \oint g^{\prime}-B \oint g \tau\right) .
$$

Proof. The instantaneous dual Pfaffian vector has the same role with the dual Darboux vector. $w$ and $w^{*}$ are respectively the Darboux vector and vectorial moment of the Darboux vector.

The instantaneous dual Pfaffian vector is

$$
\widehat{\omega}=\omega+\varepsilon \omega^{*} .
$$

Vectorial moment of Darboux vector is given by

$$
\begin{aligned}
\omega^{*} & =\alpha \wedge w \\
& =g N \wedge(\tau T+\kappa B)+\frac{g^{\prime}}{\beta} C \wedge(\tau T+\kappa B)+\left(\frac{f \tau+h \kappa}{\beta}\right) W \wedge(\tau T+\kappa B) \\
& =g \kappa T+g^{\prime} N-g \tau B
\end{aligned}
$$

If this statement is substituted in (24), the instantaneous dual Pfaffian vector is

$$
\widehat{\omega}=\tau T+\kappa B+\varepsilon\left(g \kappa T+g^{\prime} N-g \tau B\right) .
$$

Also, by taking definition of dual Steiner vector, $[5$, we can write

$$
D=\oint \widehat{\omega}=T \oint \tau+B \oint \kappa+\varepsilon\left(T \oint g \kappa+N \oint g^{\prime}-B \oint g \tau\right) .
$$


Theorem 4. The dual angles of pitch of closed ruled surfaces corresponding to $(\widehat{N}),(\widehat{C}),(\widehat{W})$ dual curves are

$$
\begin{aligned}
\Lambda_{\widehat{N}} & =\varepsilon\left(\frac{\kappa(f \tau+h \kappa)+g^{\prime} \beta \tau}{\beta^{2}} \lambda_{T}-\frac{\tau(f \tau+h \kappa)-g^{\prime} \beta \kappa}{\beta^{2}} \lambda_{B}-\oint g^{\prime}\right), \\
\Lambda_{\widehat{C}} & =\frac{1}{\beta}\left(\kappa \lambda_{T}-\tau \lambda_{B}\right)-\varepsilon\left(\frac{1}{\beta}\left(g \tau \lambda_{T}+g \kappa \lambda_{B}-\tau \oint g \tau-\kappa \oint g \kappa\right)\right), \\
\Lambda_{\widehat{W}} & =-\frac{1}{\beta}\left(\tau \lambda_{T}+\kappa \lambda_{B}\right)-\varepsilon\left(\frac{1}{\beta}\left(g \kappa \lambda_{T}-g \tau \lambda_{B}+\tau \oint g \kappa-\kappa \oint g \tau\right)\right) .
\end{aligned}
$$

Here $\lambda_{T}$ and $\lambda_{B}$ are the angles of pitch of closed ruled surfaces drawn by $T$ and $B$, respectively.

Proof. If take into account equations (3) and (23), the dual angle of pitch of closed ruled surface corresponding to the $(\widehat{N})$ dual curve is

$$
\begin{aligned}
\Lambda_{\widehat{N}}= & -\langle D, \widehat{N}\rangle=-\left\langle d+\varepsilon d^{*}, N+\varepsilon N^{*}\right\rangle \\
= & -\langle T \oint \tau+B \oint \kappa, N\rangle-\varepsilon\left[\left\langle T \oint \tau+B \oint \kappa, \frac{1}{\beta}\left((f \tau+h \kappa) C-g^{\prime} W\right)\right\rangle\right. \\
& \left.+\left\langle T \oint g \kappa+N \oint g^{\prime}-B \oint g \tau, N\right\rangle\right] \\
= & \varepsilon\left(\frac{\kappa(f \tau+h \kappa)+g^{\prime} \beta \tau}{\beta^{2}} \oint \tau-\frac{\tau(f \tau+h \kappa)-g^{\prime} \beta \kappa}{\beta^{2}} \oint \kappa-\oint g^{\prime}\right) \\
= & \varepsilon\left(\frac{\kappa(f \tau+h \kappa)+g^{\prime} \beta \tau}{\beta^{2}} \lambda_{T}-\frac{\tau(f \tau+h \kappa)-g^{\prime} \beta \kappa}{\beta^{2}} \lambda_{B}-\oint g^{\prime}\right) .
\end{aligned}
$$

Similarly, the dual angles of pitch of closed ruled surfaces corresponding to the $(\widehat{C})$ and $(\widehat{W})$ dual curves are

$$
\begin{aligned}
\Lambda_{\widehat{C}}= & -\langle D, \widehat{C}\rangle=-\left\langle d+\varepsilon d^{*}, C+\varepsilon C^{*}\right\rangle \\
= & -\langle T \oint \tau+B \oint \kappa, C\rangle-\varepsilon\left[\left\langle T \oint \tau+B \oint \kappa,-\frac{1}{\beta}((f \tau+h \kappa) N+g W)\right\rangle\right. \\
& \left.+\left\langle T \oint g \kappa+N \oint g^{\prime}-B \oint g \tau, C\right\rangle\right] \\
= & \frac{1}{\beta}(\kappa \oint \tau-\tau \oint \kappa)-\varepsilon\left(\frac{1}{\beta}(g \tau \oint \tau-\tau \oint g \tau+g \kappa \oint \kappa-\kappa \oint g \kappa)\right) \\
= & \frac{1}{\beta}\left(\kappa \lambda_{T}-\tau \lambda_{B}\right)-\varepsilon\left(\frac{1}{\beta}\left(g \tau \lambda_{T}+g \kappa \lambda_{B}-\tau \oint g \tau-\kappa \oint g \kappa\right)\right),
\end{aligned}
$$




$$
\begin{aligned}
\Lambda_{\widehat{W}}= & -\langle D, \widehat{W}\rangle=-\left\langle d+\varepsilon d^{*}, W+\varepsilon W^{*}\right\rangle \\
= & -\langle T \oint \tau+B \oint \kappa, W\rangle-\varepsilon\left[\left\langle T \oint \tau+B \oint \kappa, \frac{g^{\prime}}{\beta} N-g C\right\rangle\right. \\
& \left.+\left\langle T \oint g \kappa+N \oint g^{\prime}-B \oint g \tau, W\right\rangle\right] \\
= & -\frac{1}{\beta}(\tau \oint \tau+\kappa \oint \kappa)-\varepsilon\left(\frac{1}{\beta}(g \kappa \oint \tau-g \tau \oint \kappa+\tau \oint g \kappa-\kappa \oint g \tau)\right) \\
= & -\frac{1}{\beta}\left(\tau \lambda_{T}+\kappa \lambda_{B}\right)-\varepsilon\left(\frac{1}{\beta}\left(g \kappa \lambda_{T}-g \tau \lambda_{B}+\tau \oint g \kappa-\kappa \oint g \tau\right)\right) .
\end{aligned}
$$

Example 1. Let $\alpha(s)=\frac{1}{\sqrt{2}}(-\cos s,-\sin s, s)$ be a circular helix curve. Then, it is easy to show that

$$
\begin{aligned}
N(s) & =(\cos s, \sin s, 0), C(s)=(-\sin s, \cos s, 0), \\
W(s) & =(0,0,1), \\
\kappa(s) & =\frac{1}{\sqrt{2}}, \tau(s)=\frac{1}{\sqrt{2}} .
\end{aligned}
$$

Considering equation (2), we obtain closed ruled surfaces corresponding to the $(\widehat{N}),(\widehat{C}),(\widehat{W})$ dual curves as

$$
\begin{aligned}
\psi_{\widehat{N}}(s, v) & =N \wedge N^{*}+v N \\
& =\left(v \cos s, v \sin s, \frac{1}{\sqrt{2}} s\right), \\
\psi_{\widehat{C}}(s, v) & =C \wedge C^{*}+v C \\
& =\left(-\frac{1}{\sqrt{2}} \cos s-v \sin s, \frac{1}{\sqrt{2}} \sin s+v \cos s, \frac{s}{\sqrt{2}}\right), \\
\psi_{\widehat{W}}(s, v) & =W \wedge W^{*}+v W \\
& =\left(-\frac{1}{\sqrt{2}} \sin s,-\frac{1}{\sqrt{2}} \cos s, v\right) .
\end{aligned}
$$

These closed ruled surfaces are shown in Fig.2. Let us find the functions $f(s), g(s), h(s)$. From the equation $(13)$, we can write

$$
\begin{aligned}
-\frac{1}{\sqrt{2}} \cos s & =g(s) \cos s-g^{\prime}(s) \sin s \\
-\frac{1}{\sqrt{2}} \sin s & =g(s) \sin s+g^{\prime}(s) \cos s \\
\frac{s}{\sqrt{2}} & =f(s)+h(s) .
\end{aligned}
$$




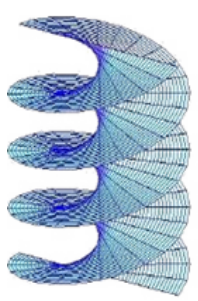

(i)

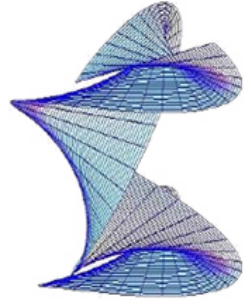

(ii)

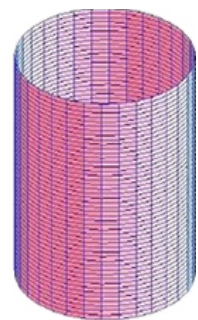

(iii)

Figure 2. The figures (i), (ii) and (iii) show closed ruled surfaces corresponding to the $(\widehat{N}),(\widehat{C})$ and $(\widehat{W})$ dual curves, respectively. Anchor curves of these surfaces are helix curve.

Using the equation (11), the solutions of $f(s), g(s), h(s)$ are given by

$$
f(s)=\frac{s}{2}, g(s)=-\frac{1}{\sqrt{2}}, h(s)=\frac{s}{2} .
$$

By taking into account the above equation and the equation (27), the dual angles of pitch of closed ruled surfaces corresponding to $(\widehat{N}),(\widehat{C}),(\widehat{W})$ dual curves are

$$
\begin{aligned}
\Lambda_{\widehat{N}} & =\varepsilon\left(\frac{s}{2}\left(\lambda_{T}-\lambda_{B}\right)-c\right), \\
\Lambda_{\widehat{C}} & =\frac{1}{\sqrt{2}}\left(\lambda_{T}-\lambda_{B}\right)-\varepsilon\left(-\frac{1}{2}\left(\lambda_{T}+\lambda_{B}\right)+\frac{1}{\sqrt{2}} \oint d s\right) \\
& =\frac{1}{\sqrt{2}}\left(\lambda_{T}-\lambda_{B}\right)-\varepsilon\left(-\frac{1}{2}\left(\lambda_{T}+\lambda_{B}\right)+\frac{1}{\sqrt{2}} L_{T}\right), \\
\Lambda_{\widehat{W}} & =-\frac{1}{\sqrt{2}}\left(\lambda_{T}+\lambda_{B}\right)-\frac{\varepsilon}{2}\left(\lambda_{B}-\lambda_{T}\right) .
\end{aligned}
$$

Here $L_{T}$ is the pitch of closed ruled surface drawn by the $T$ and $c$ is an arbitrary constant known as the integration constant.

Example 2. Let $\alpha(s)=\left(\frac{4}{5} \cos s, 1-\sin s,-\frac{3}{5} \cos s\right)$ be a curve. Then, it is easy to show that

$$
\begin{aligned}
N(s) & =\left(-\frac{4}{5} \cos s, \sin s, \frac{3}{5} \cos s\right) \\
C(s) & =\left(\frac{4}{5} \sin s, \cos s,-\frac{3}{5} \sin s\right) \\
W(s) & =\left(-\frac{3}{5}, 0,-\frac{4}{5}\right) \\
\kappa(s) & =1, \tau(s)=0 .
\end{aligned}
$$


Considering equation (2), we obtain closed ruled surfaces corresponding to $(\widehat{N}),(\widehat{C}),(\widehat{W})$ dual curves as

$$
\begin{aligned}
\psi_{\widehat{N}}(s, v) & =N \wedge N^{*}+v N \\
& =\left(\frac{4}{5} \cos s \sin s-v \frac{4}{5} \cos s, \cos ^{2} s+v \sin s,-\frac{3}{5} \cos s \sin s+v \frac{3}{5} \cos s\right), \\
\psi_{\widehat{C}}(s, v) & =C \wedge C^{*}+v C \\
& =v\left(\frac{4}{5} \sin s, \cos s,-\frac{3}{5} \sin s\right), \\
\psi_{\widehat{W}}(s, v) & =W \wedge W^{*}+v W \\
& =\left(\frac{4}{5} \cos s-v \frac{3}{5}, 1-\sin s,-\frac{3}{5} \cos s-v \frac{4}{5}\right) .
\end{aligned}
$$

These closed ruled surfaces are shown in Fig.3. Let us find the functions $f(s), g(s), h(s)$. From the equation 13 , we can write

$$
\begin{aligned}
4 \cos s & =-4 f(s) \sin s-4 g(s) \cos s-3 h(s), \\
1-\sin s & =-f(s) \cos s+g(s) \sin s, \\
-3 \cos s & =3 f(s) \sin s+3 g(s) \cos s-4 h(s) .
\end{aligned}
$$

Applying the (11), the solutions of $f(s), g(s), h(s)$ are given by

$$
f(s)=-\cos s, g(s)=\sin s-1, h(s)=0 .
$$

By taking into account the above equation and the equation (27), the dual angles of pitch of closed ruled surfaces corresponding to $(\widehat{N}),(\widehat{C}),(\widehat{W})$ are

$$
\begin{aligned}
\Lambda_{\widehat{N}} & =\varepsilon\left(\cos s \lambda_{B}-\oint \cos s d s\right) \\
\Lambda_{\widehat{C}} & =\lambda_{T}-\varepsilon\left((\sin s-1) \lambda_{B}-\oint(\sin s-1) d s\right), \\
\Lambda_{\widehat{W}} & =-\lambda_{B}-\varepsilon\left(g \lambda_{T}-c\right) .
\end{aligned}
$$

where $c$ is an arbitrary constant known as the integration constant.

Example 3. The Viviani's curve is formed by the intersection of a cylinder and a sphere. It is parametrized by

$$
\alpha(t)=\left(a(1+\cos t), a \sin t, 2 a \sin \frac{t}{2}\right) .
$$

Here, 2a is radius of sphere. The expression for the alternative invariants of Viviani's curve are given by

$$
N(s)=\left(\frac{-3-12 \cos t-\cos 2 t}{\sqrt{88 \cos t+162+6 \cos 2 t}}, \frac{-12 \sin t-\sin 2 t}{\sqrt{88 \cos t+162+6 \cos 2 t}},\right.
$$




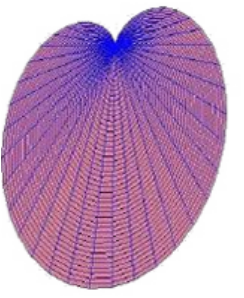

(i)

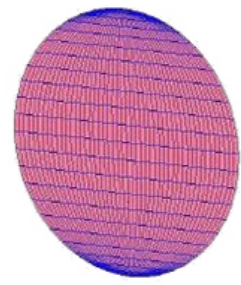

(ii)

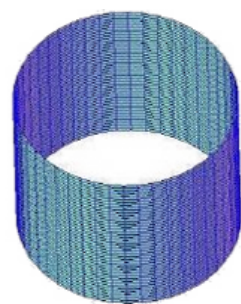

(iii)

Figure 3. The figures (i), (ii) and (iii) show closed ruled surfaces corresponding to the $(\widehat{N}),(\widehat{C})$ and $(\widehat{W})$ dual curves, respectively.

$$
\begin{aligned}
&\left.\frac{2 \sqrt{2} \sin \frac{t}{2}}{\sqrt{81+44 \cos t+3 \cos 2 t}}\right), \\
& C(s)=\quad W \wedge N \\
& {\left[\frac{\sqrt{3 \cos t+13}}{a(\cos t+3)^{3 / 2}}\left(\frac{3 \sin (t / 2)+\sin (3 t / 2)}{\sqrt{26+6 \cos t}}\right)+\frac{-6 \sqrt{2} \cos (t / 2) \sin t}{a(3 \cos t+13) \sqrt{3+\cos t}},\right.} \\
& \frac{\sqrt{3 \cos t+13}}{a(\cos t+3)^{3 / 2}}\left(\frac{-2 \sqrt{2} \cos ^{3}(t / 2)}{\sqrt{13+3 \cos t}}\right)+\frac{6 \cos (t / 2)}{a(3 \cos t+13)}\left(\frac{\sqrt{2} \cos t}{\sqrt{3+\cos t}}\right), \\
& W(s)= \frac{\left.\frac{\sqrt{3 \cos t+13}}{a(\cos t+3)^{3 / 2}}\left(\frac{2 \sqrt{2}}{\sqrt{13+3 \cos t}}\right)+\frac{6 \cos (t / 2)}{a(3 \cos t+13)}\left(\frac{\sqrt{2} \cos (t / 2)}{\sqrt{3+\cos t}}\right)\right]}{\left((3 \cos t+13) a^{-1}(\cos t+3)^{-3}+36 \cos (t / 2)^{2} a^{-2}(3 \cos t+13)^{-2}\right)}, \\
& \kappa(s)= \frac{\sqrt{3 \cos t+13}}{a(\cos t+3)^{\frac{3}{2}}}, \tau(s)=\frac{6 \cos \frac{t}{2}}{3 a \cos t+13 a} .
\end{aligned}
$$

Considering the equation 15 , closed ruled surfaces $\psi_{\widehat{N}}(s, v), \psi_{\widehat{C}}(s, v)$ and $\psi_{\widehat{W}}(s, v)$ corresponding to $(\widehat{N}),(\widehat{C})$ and $(\widehat{W})$ dual curves is plotted by using Maple program (Fig. 4). Herein, associated calculations of these surfaces are computed by Maple program.

\section{Conclusion}

In this study, the vectorial moments of the alternative vectors are written using the data in equation (13). The dual expressions of the closed ruled surfaces which corresponds to the dual curves drawn by the $\widehat{N}, \widehat{C}$ and $\widehat{W}$ on the dual sphere are expressed in terms of alternative vectors. The distribution parameters and Gauss curvatures of closed ruled surfaces $\psi_{\widehat{N}}(s, v), \psi_{\widehat{C}}(s, v)$ and $\psi_{\widehat{W}}(s, v)$, which are obtained using the equation (13), are calculated. Applying (13), likewise, it is shown that the closed ruled surface corresponding to the $(\widehat{W})$ is developable. The dual angles of pitch of these surfaces obtained using the equation $(13)$ are expressed 


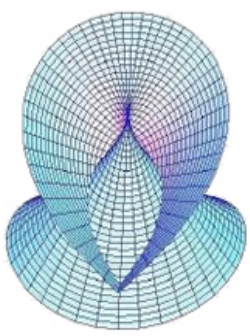

(i)

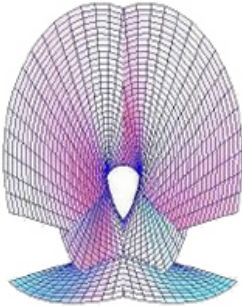

(ii)

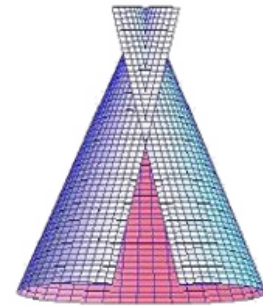

(iii)

Figure 4. The figures (i), (ii) and (iii) show closed ruled surfaces corresponding to the $(\widehat{N}),(\widehat{C})$ and $(\widehat{W})$ dual curves, respectively. Anchor curves of these surfaces are Viviani's curve.

in terms of the angles of pitch of closed ruled surfaces drawn by $T$ and $B$. Upon inspection of helix and Viviani's curves, the related ruled surfaces are generated.

\section{REFERENCES}

[1] Abbena, E., Salamon, S., Gray, A., Modern differential geometry of curves and surfaces with Mathematica, CRC press, 2005.

[2] DoCarmo M. P., Differential geometry of curves and surfaces, Prentice Hall, Englewood, Cliffs, 1976.

[3] Fenchel, W., On the Differential Geometry of Closed Space Curves, Bull. Amer. Math. Soc., 57 (1951), 44-54.

[4] Güven, İ. A., Kaya, S. and Hacısalihoğlu, H. H., On closed ruled surfaces concerned with dual Frenet and Bishop frames, J. Dyn. Syst. Geom. Theor., 9(1) (2011), 67-74.

[5] Gürsoy, O., The dual angle of pitch of a closed ruled surface, Mech. Mach. Theory, 25(2) (1990), 131-140.

[6] Gürsoy, O., On the integral invariants of a closed ruled surface, J. Geom., 39(1) (1990), $80-91$.

[7] Hacısalihoğlu, H. H., On the pitch of a closed ruled surface, Mech. Mach. Theory, 7(3) (1972), 291-305.

[8] Huyghens, C., Horologium ascillatorium, part III, 1963.

[9] Kasap, E. and Kuruoğlu, N., The Bertrand offsets of ruled surfaces in $\mathbb{R}_{1}^{3}$, Acta Math Vietnam, 31 (2006), 39-48.

[10] Kasap, E., Yüce, S. and Kuruoğlu, N., The involute-evolute offsets of ruled surfaces, Iranian J. Sci. Tech. Transaction A, 33 (2009), 195-201.

[11] Kaya, O. and Önder, M., New Partner Curves in The Euclidean 3-Space $E^{3}$, Int. J. Geom., $6(2)$ (2017), 41-50.

[12] Liu, H. and Wang, F., Mannheim partner curves in 3-space, Journal of Geometry, 88(1-2) (2008), 120-126.

[13] O'neill, B., Elemantary Differential Geometry, Second Edition, Elsevier, 1996.

[14] Orbay, K., Kasap, E. and Aydemir. İ., Mannheim offsets of ruled surfaces, Math Problems Engineering, Article ID 160917 (2009), 9 pages.

[15] Pears, L. R., Bertrand curves in Riemannian space, J. London Math. Soc., 1-10(2) (1935), $180-183$. 
[16] Ravani, B. and Ku, T. S., Bertrand offsets of ruled and developable surfaces, Computer-Aided Design, 23(2) (1991), 145-152.

[17] Rashad, A. Abdel-Baky, An explicit characterization of dual spherical curve, Commun. Fac. Sci. Univ. Ank. Series A, 51(2) (2002), 43-49.

[18] Tunçer, Y., Vectorial moments of curves in Euclidean 3-space, International Journal of Geometric Methods in Modern Physics, 14(02) (2017), 1750020.

[19] Uzunoğlu, B., Gök İ. and Yaylı, Y., A new approach on curves of constant precession, Appl. Math. Comput., 275 (2016), 317-232.

[20] Yayl, Y. and Saraçoğlu, S., Some Notes on Dual Spherical Curves, Journal of Informatics and Mathematical Sciences, 3(2) (2011), 177-189.

Current address: Süleyman Şenyurt (Corresponding author): Faculty of Arts and Sciences, Department of Mathematics, Ordu Universty, Ordu, Turkey.

E-mail address: senyurtsuleyman@hotmail.com

ORCID Address: http://orcid.org/0000-0003-1097-5541

Current address: Abdussamet Çalışkan: Faculty of Arts and Sciences, Department of Mathematics, Ordu Universty, Ordu, Turkey.

E-mail address: abdussamet65@gmail.com

ORCID Address: http://orcid.org/0000-0002-1512-2452 